\title{
The Construction of Carbon Nanotubes Containing an Anti-Bacterial Chemical Component and its Effect on MDR and XDR Isolates of Pseudomonas Aeruginosa
}

\author{
Kamelia Banihashemi ${ }^{1}$, Behnam Sobouti ${ }^{2}$, Iraj Mehregan ${ }^{1}$, \\ Ronak Bakhtiari ${ }^{3}$, Nour Amirmozafari*4
}

\begin{abstract}
Background: Pseudomonas aeruginosa is an opportunistic human pathogen that causes severe acute and chronic nosocomial infections, especially in immunocompromised burn patients. and can lead to severe mortality and morbidity. The emergence of antibiotic resistant $P$. aeruginosa infections has created significant challenges in treating these patients. A potential alternative treatment for antibiotic resistant pathogens includes the use of carbon nanotubes (CNTs), which have received considerable attention due to their potent antibacterial activity. The aim of this study was to construct a novel CNT containing an anti-bacterial chemical component to effectively combat drug resistant $P$. aeruginosa infections.

Methods: In this study, a novel chemical component was synthesized and coated the CNT. The antimicrobial effects were then evaluated on MDR, XDR, and PDR strains of $P$. aeruginosa isolated from burn patients. Antibiotic susceptibility was evaluated using the disk diffusion test and minimum inhibitory concentration (MIC) testing. In order to determine the potential cytotoxicity, an MTT assay was performed on Human Dermal Fibroblasts. The effect of treatment on the expression of wound healing genes was analyzed via qRT-PCR.

Results: Experimental data indicates that our CNT coated chemical compound had antibacterial properties, negligible cytotoxicity, and could accelerate the wound healing process.

Conclusions: Given the antibacterial properties of our CNT chemical compound, it has the potential to treat and reduce the occurrence of multi-drug resistant $P$. aeruginosa burn wound infections and aid in wound healing by turning on genes (VEGFA, EGF and PDEGF) involved in the wound healing process.
\end{abstract}

Keywords: Anti-Bacterial drugs, Drug resistance, Nanotubes Carbon, Pseudomonas aeruginosa, MDR, XDR.

\section{Introduction}

Pseudomonas aeruginosa is an opportunistic pathogen involved in the development of severe acute and chronic healthcare-associated infections (HAIs). Immunocompromised burn patients are particularly vulnerable to the development of $P$. aeruginosa wound infections causing significant morbidity and mortality $(1,2)$. The emergence of antibiotic resistance of $P$. aeruginosa to imipenem, quinolones and third-generation cephalosporins has increased to 15,9 and $20 \%$, respectively $(3,4)$. Most clinical isolates of $P$. aeruginosa show resistance to at least three different classes of antibiotics including, aminoglycosides, anti-plasma penicillins, 
cephalosporins, carbapenems and fluoroquinolones (5). P. aeruginosa is categorized into different phenotypes based on the different drug resistance patterns including: 1) multidrugresistant (MDR), bacteria that show resistance to several antibiotics, 2) extensively drug-resistant (XDR), bacteria that have acquired resistance due to prolonged antibiotic exposure, 3) pandrugresistant (PDR), bacteria resistant to all current antibacterial agents $(6,7)$.

Nanotechnology has contributed to the development of novel antibacterial biomaterials. Among the nanomaterials that have emerged, carbon nanotubes (CNTs) have received considerable attention for their potent antibacterial activity due to the unique biological, physical and chemical properties. The diameter of carbon nanotubes is roughly $1-3 \mathrm{~nm}$ and significantly contributes to its antibacterial activity. This small diameter enables the CNT to induce damage within the bacterial cell membrane through cellsurface interactions $(8,9)$. Combining CNTs with different chemicals can lead to increased efficacy and new therapeutic potential. Although mercury $(\mathrm{Hg})$ is a potentially toxic element, its potent antibacterial properties has led to its widespread use for infection control $(10,11)$. In this study, a new combination of $\mathrm{Hg}$ and CNTs was created to act as an effective antibacterial agent against the MDR, XDR, and PDR strains of P. aeruginosa. The effect of our CNT on wound healing was measured by determining the expression of wound healing-associated genes in vitro.

\section{Materials and methods \\ Participants}

This prospective, observational study was conducted at Motahari hospital, Tehran from January to December 2018. Burn victim patients with a known diagnosis of $P$. aeruginosa wound infections were included in the study. All patients gave their written consent for participation in this study.

\section{Collection and processing of specimens}

Burn wound specimens were collected from patients by swabbing the burned skin area. A total of 122 clinical specimens were collected. The sampled swabs were initially placed in Thioglycolate broth tubes and incubated for overnight. Following overnight incubation, the broths were sub-cultured onto blood agar and Eosin Methylene Blue plates and incubated for any bacterial colonies to appear. All bacterial colonies were processed for identification and antibiotic susceptibility testing in compliance with the Clinical and Laboratory Standard Institute (CLSI) guidelines 2014 (12). A total of 72 P. aeruginosa strains were isolated.

\section{Identification and antibiotic susceptibility}

Pseudomonas aeruginosa was identified by its Gram-negative characteristic, oxidase positivity, pigment production, colony characteristics, grape-like odor, motility, ability to decarboxylate arginine, and growth at $42{ }^{\circ} \mathrm{C}$ (13). Polymerase Chain Reaction (PCR) was used as molecular confirmation for the presence $P$. aeruginosa. Specific primers including,

ATGGAAATGCTGAAATTCGGC as the forward primer and TTCTTCAGCTCGACGCGACG as the reverse primer were used (14). Antibiotic sensitivity patterns of these isolates were determined by disk diffusion method using the CLSI 2014 guidelines (15). The susceptibility patterns of all tested antibiotics are listed in Table 1.

\section{Preparation of chemical component}

1-Methyl-1,2,3,4-tetrazole-5-thiol was purchased from Sigma Aldrich Co. Chemicals including, ethylene diamine (99\%), mercury iodide II (99\%), dimethyl sulfoxide (99\%), and methanol $(99.6 \%)$ were purchased from Merck Co. All materials were used without additional purification. Infrared spectroscopy (4000e250 $\mathrm{cm} 1)$ of solid specimens were taken as $1 \%$ dispersion in CsI pellets using a Shimadzu-470 spectrometer at $300.13 \mathrm{MHz}$ in DMSO-d6 protons (Bruker AC-300) as used for registration of NMR spectra data. Kofler Heizbank Rechart (type 7841) were used for determining melting points. Heraeus $\mathrm{CHNeO}$ Rapid analyzer was used for elemental analysis. STA 503 B€ ahr apparatus was used for analysis of thermal behavior (16). 
Preparation of Carbon nanotube (CNT) containing chemical component

The nanoprecipitation method was used to prepare the CNT containing chemical component as previously described (17). Briefly, $125 \mathrm{mg}$ of CNT was dispersed in deionized water (in $20 \mathrm{~mL}$ ) by magnetic stirring for $30 \mathrm{~min}$ at $60{ }^{\circ} \mathrm{C}$. Following the stirring, 0.4 $\mathrm{g}$ of pluronic F-127 suspension was added to the solution. Then $20 \mathrm{~mL}$ of glutaraldehyde (5\% $\mathrm{v} / \mathrm{v})$ was added. After $24 \mathrm{~h}$ of crosslinking, the solution was then centrifuged at $15000 \mathrm{~g}$ for 30 min at a temperature of $60{ }^{\circ} \mathrm{C}$ to improve redispersion in water and remove any unreacted glutaraldehyde. CNTs were frozen by liquid nitrogen and freeze-dried for $40 \mathrm{~h}$ (FDB5503, Operon, Gimpo, South Korea).

Loading the chemical component in the CNTs was achieved using the incubation method. Briefly, $500 \mathrm{mg}$ of the dried nanoparticle was added into a dialysis membrane and allowed to swell in deionized water for $1 \mathrm{~h}$ with continuous magnetic stirring at a temperature of $37{ }^{\circ} \mathrm{C}$. The saturated chemical component was mixed with the soaked nanoparticle, followed by continued stirring for $24 \mathrm{~h}$. The final preparation was then dried in a dry oven.

\section{Cell viability assay}

To measure the potential cytotoxicity of the CNT and determine optimum doses, the 3- (4, 5-dimethylthiazol-2-yl) $\quad-2, \quad 5-$ diphenyltetrazolium bromide (MTT) assay was used according to Van Meerloo experiment (18). Human Dermal Fibroblasts cells (HDFa) were seeded in 96-well plates in $100 \mu \mathrm{l}$ of RPMI medium at a density of $1 \times 10^{4}$ cells/well. Twenty-four hours after seeding, the medium was discarded and fresh RPMI medium was added. The wells were then loaded with chemical component $\left(\left\{\left[\mathrm{H}_{2} \mathrm{en}\right]\left[\mathrm{Hg}_{2}(\mathrm{mmtz})_{4}(\mu-\right.\right.\right.$ $\left.\left.\left.\mathrm{I})_{2}\right]\right\}\right)$ alone and with CNT coated with the chemical component ranging from 5, 10, 20, 50, 75,150 and $300 \mu \mathrm{g} / \mathrm{ml}$. After $24 \mathrm{~h}$ of incubation, MTT solution was added into each well and incubated for $4 \mathrm{~h}$ in a $\mathrm{CO}_{2}$ incubator at $37{ }^{\circ} \mathrm{C}$. Lastly, the optical density of purple formazan absorbance was determined at $570 \mathrm{~nm}$ by a microplate reader (Bio-Tek Instruments Inc., Vermont, USA). Cell viability were calculated using the formula, \% viability $=($ optical density of sample/optical den sity of control) $\times 100$.

\section{Determining mRNA levels by $q R T-P C R$ in mouse model}

Rats (Male Wistar strain of 6-8 weeks of age) were separated into five different treatment groups: control, burn skin rats treated with the CNTs coated chemical component $\left(\left\{\left[\mathrm{H}_{2} \mathrm{en}\right]\left[\mathrm{Hg}_{2}(\mathrm{mmtz})_{4}(\mu-\mathrm{I})_{2}\right]\right\}\right)$, burn skin rats treated with the chemical component and infected with $P$. aeruginosa PAO1 strain, burn skin rats infected with PAO1 strain, and burn skin rats treated with the chemical component and infected with the PAO1 strain. This final group received an injection of meropenem as a pre-treatment.

RNA was extracted from rat tissue skin samples (epidermis, basement membrane, hair follicles, and dermis) using the RNeasy Mini Kit (50) (Qiagen, Germany), according to the manufacturer's protocol. The extracted RNA was stored at $-70{ }^{\circ} \mathrm{C}$ prior to use. RNA integrity, quantity, and quality were determined by agarose gel electrophoresis and Nano Drop system (Nano Drop Technologies, Inc., Wilmington, DE, USA). One $\mu \mathrm{g}$ samples of RNA were used for complementary DNA (cDNA), which was synthesized using the total Reverse Transcription Kit (Takara, Japan) after adjustment of the concentrations, according to the manufacturer's instructions. The beta-actin gene was used as the reference gene. The sequences of the primers used are listed in Table 1. qPCR was carried out using a real-time PCR cycler (Rotor-Gene Q MDx, Qiagen).

\section{Statistical analysis}

Statistical analyse was performed with REST 2009 as well as Excel 2019. 
Table 1. All primers used in this study.

\begin{tabular}{lllllllll}
\hline \multicolumn{1}{c}{ Name } & \multicolumn{1}{c}{ Sequence (5-3) } & MW & $\begin{array}{c}\text { OD } \\
(\mathbf{1 0 0 0} \boldsymbol{\mu})\end{array}$ & $\begin{array}{c}\text { nmol } \\
\text { EGF.F }\end{array}$ & $\begin{array}{c}\text { water/tube } \\
(\boldsymbol{\mu l})\end{array}$ & TM & GC\% & Mer \\
\hline EGF.R & CGGTGCCTTGCCCTGACTCTAC & 6622.3 & 3 & 14.95 & 149.50 & 63.98 & 59.1 & 22 \\
PDEGF.F & TCTTCCTGCCTCTCTTCTCTCCAA & 6686.3 & 2.5 & 12.34 & 123.39 & 62.12 & 54.5 & 22 \\
PDEGF.R & CCATCTTCGTCTACGGAGTCTCTG & 6579.2 & 2.5 & 12.54 & 125.39 & 62.12 & 54.5 & 22 \\
VEGFA.F & GAAGTGGTGAAGTTCATGGAC & 6550.7 & 3 & 13.62 & 136.16 & 64.43 & 54.2 & 24 \\
VEGFA.R & GCATGATCTGCATAGTGACG & 6157 & 2.5 & 10.08 & 100.76 & 54.87 & 47.6 & 21 \\
\hline
\end{tabular}

\section{Results}

\section{Antimicrobial susceptibility of $P$. aeruginosa}

Molecular identification of the $P$. aeruginosa isolates was performed by $\mathrm{PCR}$ using specific primers. The amplicon size was 503 bp. The findings are presented in Figure Fig. 1.

Antimicrobial susceptibility patterns of the $P$. aeruginosa isolates are shown in Table 2 . The results indicate that $74 \%$ and $26 \%$ of the isolates were classified as MDR and XDR strains, respectively. Moreover, the MIC of the CNTs, the chemical component and the coated CNT were determined to be 2048,128 and $64 \mu \mathrm{l} / \mathrm{ml}$, respectively.

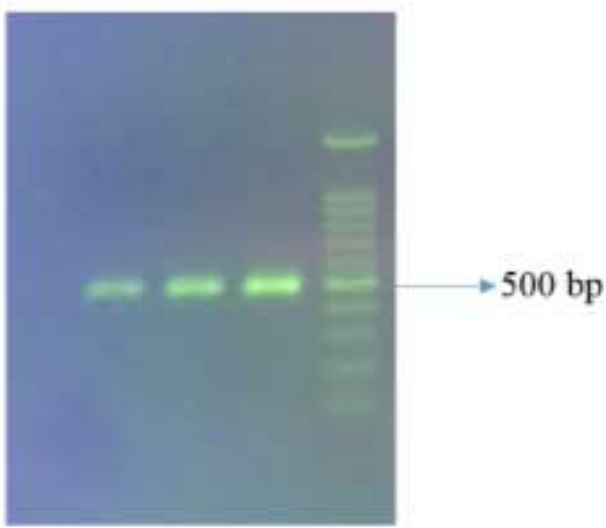

Fig. 1. The molecular identification of $P$. aeruginosa isolates.

Table 2. The percent overall resistant rate of the Pseudomonas aeruginosa isolates against the tested antibiotics.

\begin{tabular}{ll}
\hline Antibiotics & Resistance rate $(\mathbf{n}, \mathbf{\%})$ \\
\hline Colistin & $6(8.33)$ \\
Aztreonam & $33(45.8)$ \\
Gentamicin & $71(98.6)$ \\
Meropenem & $65(90.27)$ \\
Levofloxacin & $72(100)$ \\
Fosfomycin & $13(18.05)$ \\
Piperacillin+ Tazobactam & $39(51.16)$ \\
\hline
\end{tabular}

\section{IC50 detection of CNT and coated CNT}

The viability of $P$. aeruginosa cells after treatment with the chemical component and coated CNT were analyzed by MTT staining. The IC50 of the chemical component and the loaded CNT were 1482.54 and $379.19 \mu \mathrm{g} / \mathrm{ml}$, respectively. The effect of the chemical component and the loaded CNT on $P$. aeruginosa at various concentrations are shown in Figures 2.

\section{Wound healing assay by $q R T-P C R$}

In order to identify differential gene expression, 3 genes (VEGFA, EGF and PDEGF) involved in wound healing were examined and their expression 
levels were determined by qRT-PCR. Comparison between group 1 and 2 showed the efficiency of the chemical component on wound healing. To determine the effect of $P$. aeruginosa infection on the expression of wound healing genes, group 1 and 4 were compared. All three genes were up- regulated in the groups that received treatment with the

A

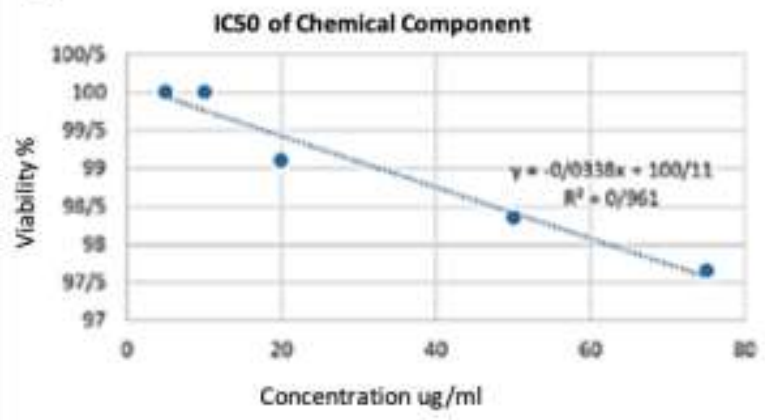

chemical component. These findings are shown in Figure 3.

Bacterial infection was observed to drastically down-regulate the expression of the wound healing genes within the rat tissue (epidermis, basement membrane, hair follicles, and dermis). Colony counts were performed in all groups; the results are presented in Table 3.

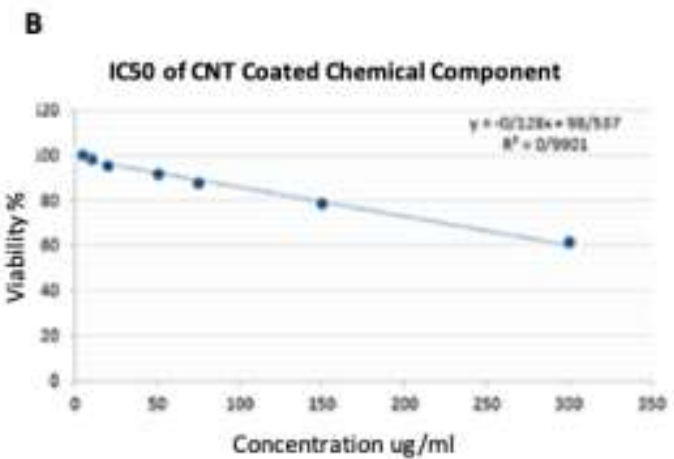

Fig. 2. Various concentrations of chemical component $\left(\left\{\left[\mathrm{H}_{2} \mathrm{en}\right]\left[\mathrm{Hg}_{2}(\mathrm{mmtz})_{4}(\mu-\mathrm{I})_{2}\right]\right\}\right)$ and its loaded CNT on Pseudomonas aeruginosa , (A) MTT diagram of the chemical compound toxicity, (B) MTT diagram of chemical compound with carbon nanotubes toxicity.

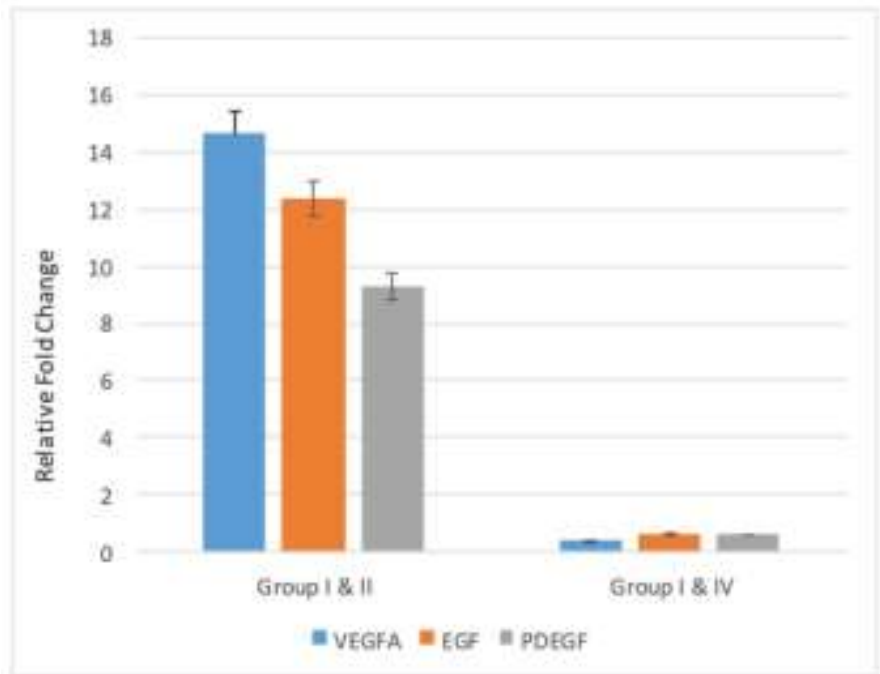

Fig. 3. Relative expression of VEGFA, EGF and PDEGF in rat tissues compared with categorized group. * $\mathrm{p}<0.05, * * \mathrm{p}<0.001$.

Table 3. Bacterial counts in the five groups.

\begin{tabular}{cl}
\hline Group & P. aeruginosa \\
\hline 1 & 717 CFU/gr (Total count) \\
2 & No growth \\
3 & 12 CFU/gr (P. aeruginosa) \\
4 & $5.4 X 10^{\wedge} 7$ CFU/gr $($ P. aeruginosa $)$ \\
5 & No growth \\
\hline
\end{tabular}

\section{Discussion}

Our findings show a high prevalence of antibiotic resistant $P$. aeruginosa isolates found in the wounds of hospitalized burn patients. Of these isolates, $51 \%$ were found to be resistant to piperacillin, which is less than a previous report showing piperacillin resistance in $73 \%$ of isolates 
(13). The resistance to beta-lactams among $P$. aeruginosa strains have been found to pose a serious threat. Of the beta-lactam class of antibiotics, the resistance is mainly against the third- and fourth-generation cephalosporins. Several molecular mechanisms have been proposed to be responsible for resistance to these antibiotics, such as the presence of bla genes in integrons, the generation of extended spectrum beta-lactamases (ESBL) enzymes, failure of porin genes to increase their expression level and/or variation of antibiotic target sites (19). Third- and fourth-generation cephalosporins with antipseudomonal activity include Ceftazidime and Cefepime, respectively. According to a recent investigation, the percentage of resistance towards cephalosporin group of antibiotics was $67.86 \%$ for Ceftazidime, $94.64 \%$ for Cephalexin and $69.64 \%$ for Cefepime (13).

Our study showed $100 \%$ of the $P$. aeruginosa isolates to be resistant to levofloxacin, $98.6 \%$ were resistant to gentamicin, and $90.27 \%$ were resistant to meropenem. Previous studies from other countries observed a similar trend in $P$. aeruginosa antibiotic resistance and some had reported $100 \%$ resistance against fluoroquinolones $(13,21)$. This points to the declining efficacy of fluoroquinolones as an effective antibiotic in controlling nosocomial Pseudomonas infections. Fluoroquinolones are an important class of antibiotics used for a variety of infections. New generation fluoroquinolones are effective against Gram-negative and Grampositive bacteria, however older fluoroquinolones are mostly effective against aerobic Gramnegative bacteria (22). Our data showed $8.33 \%$ of the $P$. aeruginosa isolates to be resistant to colistin and $18.05 \%$ showed resistance to Fosfomycin.

Aminoglycosides are potent broad-spectrum antibiotics, containing a unique aminocyclitol ring. They are highly effective against aerobic and facultative aerobic Gram-negative bacteria (23). We detected $98.6 \%$ resistance towards gentamicin in this study which was twice as those reported by Panayotis T. Tassios et al (24). Since the previous study was conducted in 1998, the sharp rise in gentamicin resistance may be due to increased usage of this antibiotic overtime.

Resistance to Aztreonam was detected in $45.8 \%$ of our $P$. aeruginosa isolates. Most of these isolates were sensitive to Fosfomycin and Colistin. Similar findings were reported from other countries with a high prevalence of $P$. aeruginosa infections (25).

In a study by Palavutitotai et al, 255 patients with $P$. aeruginosa infections admitted to Siriraj Hospital in Bangkok, Thailand were examined for antibiotic resistance. Of the participants, $56(22 \%)$ cases showed an XDR phenotype, 32 (12.5\%) were MDR, and 167 (65.5\%) were reported as non-MDR. It should be noted that their findings also showed $P$. aeruginosa isolates to be resistant to carbapenem antibiotics (16). Our findings show that $19 \%$ of $P$. aeruginosa infections have an XDR phenotype and $53 \%$ have a MDR phenotype. In addition, no PDR strains were found in our study, nor in the work by Palavutitotai et al.

To our knowledge, this is the first study from Iran that shows the prevalence of MDR, XDR, and PDR $P$. aeruginosa infections among hospitalized burn patients. The strengths of this study include the development of a novel chemical component effective against antibiotic resistant $P$. aeruginosa infections that also aids in the induction of wound healing-associated genes. Limitation of the current study was that we have not yet tested the chemical compound directly on human burn wounds.

Drug resistant strains of $P$. aeruginosa are responsible for serious nosocomial infections, especially among hospitalized burn patients. Our CNT coated biomaterial could help to prevent and treat wound infections, and support wound healing by activating genes (VEGFA, EGF and PDEGF) involved in wound healing.

\section{Acknowledgment}

We would like to thank all patients who participated in this study. This article is the result of Kamelia Banihashemi's Ph. D thesis project. The authors declare no conflict of interest. 


\section{References}

1. Pollack M. Pseudomonas aeruginosa. Principles and Practice of Infectious Diseases. 4th ed. New York: Churchill Livingstone, 1995:1980-2003.

2. Imai E, Ueda M, Kanao K, Miyaki K, Kubota T, Kitajima M. Surgical site infection surveillance after open gastrectomy and risk factors for surgical site infection. J Infect Chemother. 2005;11(3):141-5.

3. Nicastri E, Petrosillo N, Martini L, Larosa M, Gesu G.P, Ippolito G. Prevalence of nosocomial infections in 15 Italian hospitals: first point prevalance study for the INF-NOS project. Infection. 2003;31 Suppl 2:10-5.

4. Carmeli Y, Troillet N, Eliopoulos GM, Samore MH. Emergence of antibiotic-resistant Pseudomonas aeruginosa: comparison of risks associated with different antipseudomonal agents. Antimicrob Agents Chemother. 1999;43(6):1379-82.

5. Magiorakos A.P, Srinivasan A, Carey RB, Carmeli Y, Falagas M.E, Giske C.G, et al. Multidrug-resistant, Extensively Drug-Resistant and Pandrug-Resistant Bacteria: An International Expert Proposal for Interim Standard Definitions for Acquired Resistance. Clin Microbiol Infect. 2012:18(3):268-81.

6. Davies J, Davies D. Origins and evolution of antibiotic resistance. Microbiol Mol Biol Rev. 2010;74(3):417-33.

7. Wardhana A, Djan R, Halim Z, disasters f. Bacterial and antimicrobial susceptibility profile and the prevalence of sepsis among burn patients at the burn unit of Cipto Mangunkusumo Hospital. 2017;30(2):107-115.

8. Cambre S, Campo J, Botka B, van Werveke W, Obrzut J, Wenseleers W, et al. Controlling the Inner Dielectric Environment of Carbon Nanotubes to Tune Their Optical Properties. The Electrochemical Society. 235th ECS Meeting 2019; 9:699-699.

9. Saliev T. The advances in biomedical applications of carbon nanotubes. Journal of Carbon Research. 2019;5(2):29.

10. Thorpe TC, Wilson M.L, Turner J.E, DiGuiseppi J.L, Willert M, Mirrett S, et al. BacT/Alert: an automated colorimetric microbial detection system. J clin microbiol. 1990;28(7):1608-12.

11. Sader HS, Castanheira M, Mendes RE, Flamm RK, Farrell DJ, Jones RNJAa, et al. Ceftazidime-avibactam activity against multidrug-resistant Pseudomonas aeruginosa isolated in US medical centers in 2012 and 2013. Indian Journal of Pathology and Microbiology. 2015;59(6):3656-3659.

12. Mundhada AS, Tenpe S. A study of organisms causing surgical site infections and their antimicrobial susceptibility in a tertiary care government hospital. 2015;58(2):195-200.

13.Javiya VA, Ghatak SB, Patel KR, Patel J. Antibiotic susceptibility patterns of Pseudomonas aeruginosa at a tertiary care hospital in Gujarat, India. 2008;40(5):230-234.

14. Taheriha M, Ghadermazi M, Amani VJJoMS. Dimeric and polymeric mercury (II) complexes of 1-methyl-1, 2, 3, 4-tetrazole-5-thiol: Synthesis, crystal structure, spectroscopic characterization, and thermal analyses. 2016;1107:57-65.

15. Collee J, Duguid J, Fraser A, Marmion B, Simmons A, Marimion B. Laboratory strategy in the diagnosis of infective syndromes. New York: Churchill Livingstone; 2006.

16. Palavutitotai N, Jitmuang A, Tongsai S, Kiratisin P, Angkasekwinai N. Epidemiology and risk factors of extensively drug-resistant Pseudomonas aeruginosa infections. 2018;13(2):e0193431.

17. Boguslavsky Y, Fadida T, Talyosef Y, Lellouche J.P. Controlling the wettability properties of polyester fibers using grafted functional nanomaterials. Journal of Materials Chemistry. 2011;21(28):10304-10310.

18. Van Meerloo J, Kaspers GJ, Cloos J. Cell sensitivity assays: the MTT assay. Methods Mol Biol. 2011;731:237-45.

19. Pfeifer Y, Cullik A, Witte W. Resistance to cephalosporins and carbapenems in Gram-negative bacterial pathogens. Int $\mathrm{J}$ Med Microbiol. 2010;300(6):371-9.

20. Rodriguez-Martinez J.M, Poirel L, Nordmann P. Molecular epidemiology and mechanisms of carbapenem resistance in Pseudomonas aeruginosa. Antimicrobial agents chemotherapy. 2009;53(11):4783-8. 
21. Hooper DC. Mechanisms of action and resistance of older and newer fluoroquinolones. Clin Infect Dis. 2000;31Supple 2:S24-8.

22. Kim J, Kang CI, Baek JY, Cho SY, Kim SH, Ko KS, et al. Treatment failure due to induction of ciprofloxacin resistance during combination therapy with colistin and ciprofloxacin in multidrug-resistant Pseudomonas aeruginosa bacteraemia. International journal of antimicrobial agents. 2014;43(4):391.

23. Kettner M, Kallova J, Hletkova M, Milosovic

$\mathrm{P}$. Incidence and mechanisms of aminoglycoside resistance in Pseudomonas aeruginosa serotype O11 isolates. Infection. 1995;23(6):380-3.

24. Tassios PT, Gennimata V, Maniatis AN, Fock C, Legakis NJ, Group GPaS. Emergence of multidrug resistance in ubiquitous and dominant Pseudomonas aeruginosa serogroup O: 11. The Greek Pseudomonas Aeruginosa Study Group. J clin microbiol. 1998;36(4):897-901.

25 . Chatterjee S. Study of some genetic markers in some ethnic groups of Indian population. University of Kalyani.2013. 